\title{
Disease-modifying polymorphisms and C609Y mutation of RET associated with high penetrance of phaeochromocytoma and low rate of MTC in MEN2A
}

\author{
Rowena Speak', Jackie Cook4, Barney Harrison ${ }^{3}$ and John Newell-Price, ${ }^{1,2}$ \\ 1Departments of Oncology and Metabolism, University of Sheffield, ${ }^{2}$ Endocrinology and ${ }^{3}$ Endocrine Surgery, \\ Royal Hallamshire Hospital, Sheffield Teaching Hospitals NHS Foundation Trust, Sheffield, UK, and \\ ${ }^{4}$ Department of Genetics, Sheffield Children's Hospital, Sheffield, UK
}

Correspondence should be addressed to J Newell-Price Email

j.newellprice@sheffield.ac.uk

\section{Summary}

Mutations of the rearranged during transfection (RET) proto-oncogene, located on chromosome 10q11.2, cause multiple endocrine neoplasia type 2A (MEN2A). Patients with mutations at the codon 609 usually exhibit a high penetrance of medullary thyroid cancer (MTC), but a sufficiently low penetrance of phaeochromocytoma that screening for this latter complication has been called to question. Patients with other RET mutations are at higher risk of younger age onset phaeochromocytoma if they also possess other RET polymorphisms (L769L, S836S, G691S and S904S), but there are no similar data for patients with 609 mutations. We investigated the unusual phenotypic presentation in a family with MEN2A due to a C609Y mutation in RET. Sanger sequencing of the entire RET-coding region and exon-intron boundaries was performed. Five family members were $\mathrm{C609Y}$ mutation positive: $3 / 5$ initially presented with phaeochromocytoma, but only $1 / 5$ had MTC. The index case aged 73 years had no evidence of MTC, but presented with phaeochromocytoma. Family members also possessed the G691S and S904S RET polymorphisms. We illustrate a high penetrance of phaeochromocytoma and low penetrance of MTC in patients with a RET C609Y mutation and polymorphisms G691S and S904S. These data highlight the need for life-long screening for the complications of MEN2A in these patients and support the role for the screening of RET polymorphisms for the purposes of risk stratification.

\section{Learning points:}

- C609Y RET mutations may be associated with a life-long risk of phaeochromocytoma indicating the importance of life-long screening for this condition in patients with MEN2A.

- C609Y RET mutations may be associated with a lower risk of MTC than often quoted, questioning the need for early prophylactic thyroid surgery discussion at the age of 5 years.

- There may be a role for the routine screening of RET polymorphisms, and this is greatly facilitated by the increasing ease of access to next-generation sequencing.

\section{Background}

Multiple endocrine neoplasia type 2A (MEN2A) is an autosomal dominant syndrome caused by mutations in the rearranged during transfection (RET) proto- oncogene located on chromosome 10q11.21 (http:// www.omim.org/entry/164761, 27.7.2016). Those affected have a predisposition to develop medullary 


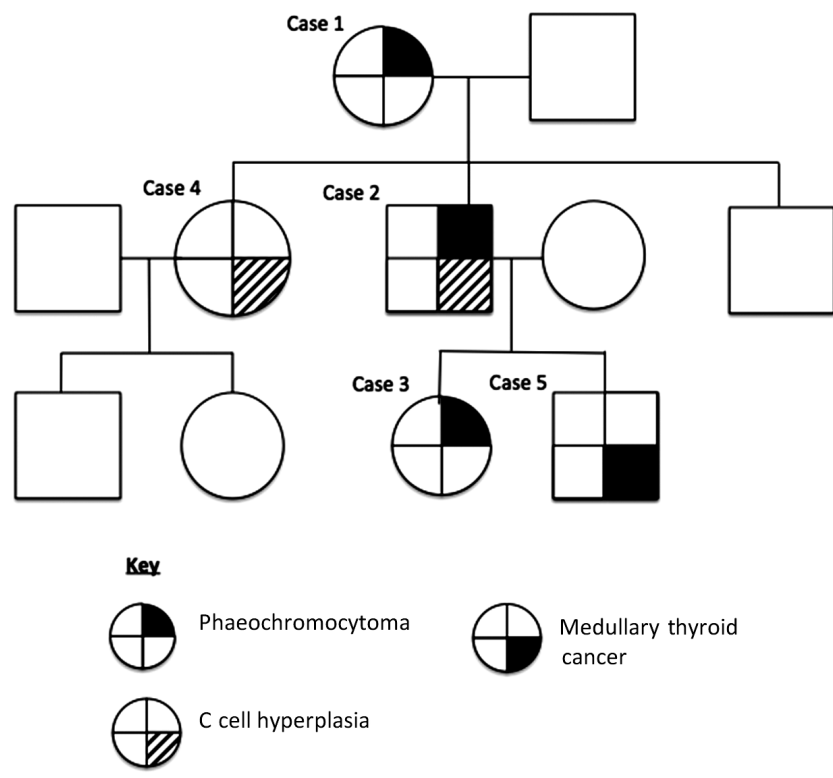

Figure 1

Family tree depicting penetrance of MEN2A.

thyroid cancer (MTC), phaeochromocytoma and hyperparathyroidism. Mutations in numerous codons of the RET proto-oncogene are associated with the development of MEN2A. The penetrance of MTC and phaeochromocytoma varies according to which codon carries the mutation and, more specifically, by the different amino acid substitutions at each codon (1). There are further data to suggest that possession of two or more RET polymorphisms L769L, S836S, G691S and S904S in those with some RET disease-associated codon mutations can increase the likelihood of developing phaeochromocytoma, with presentation at a younger age (2), but there are no such data for those with mutations at codon 609. Moreover, there are conflicting data as to the penetrance of phaeochromocytoma in patients with codon 609 mutations (3), and it has even been questioned whether there is a need to screen for phaeochromocytoma in these patients (1). Here, our objective was to investigate an unusually high penetrance of phaeochromocytoma and low penetrance of MTC across three generations in a family with the C609Y mutation (Figure 1).

\section{Case presentation}

\section{Case 1}

A 73-year-old Caucasian female presented to our institution in 2009 with a history of recurrent dizziness and shaking over several years.
Case 2

Case 1's son, a 54-year-old man, had a left-sided phaeochromocytoma in 1988 aged 27 (before genetic testing was available) and underwent an adrenalectomy at that time at another institution.

\section{Case 3}

Case 3 is the daughter of Case 2, and hence, granddaughter of Case 1. She was admitted to hospital in 2008, aged 25 , after a 2-year history of worsening abdominal pain, headaches and weight loss.

\section{Case 4}

Case 4 is the daughter of Case 1, and sister of Case 2. After the positive test for the C609Y mutation in her mother and brother, she tested positive for this mutation in 2009, aged 50 years.

\section{Case 5}

Case 5 is the son of Case 2 and brother of Case 3. In 2009, aged 23 years, after detection of the C609Y RET mutation in his grandmother and father, this patient was found to carry the same mutation.

\section{Investigation}

\section{Case 1}

Blood pressure was $140 / 67 \mathrm{mmHg}$. 24-h urinary metadrenaline was $2326-3249 \mathrm{nmol} / 24 \mathrm{~h}(0-2000)$. MRI revealed a heterogenous right adrenal lesion $2.5 \mathrm{~cm}$ in diameter, with high signal on $\mathrm{T} 2$ sequences, and which showed uptake on MIBG scintigraphy, confirming a diagnosis of phaeochromocytoma. Basal parathyroid hormone and calcitonin were modestly raised at $11.1 \mathrm{pmol} / \mathrm{L}(1.5-6.9)$ and $30.1 \mathrm{ng} / \mathrm{L}(0-5.5)$ respectively, with adjusted serum calcium just above normal range at $2.7 \mathrm{mmol} / \mathrm{L}$ (2.20-2.60). Ultrasound of the thyroid found suspicious lesions bilaterally. However, fine needle aspiration biopsies confirmed these to be benign nodules.

\section{Case 2}

After the diagnosis of phaeochromocytoma in his mother in 2009, Case 2 underwent genetic testing, which showed that he had the C609Y mutation in the RET proto-oncogene. Plasma calcitonin rose from a basal 
value of $4.5 \mathrm{ng} / \mathrm{L}$ to a peak value of $90.9 \mathrm{ng} / \mathrm{L}$ (0-18.9) after pentagastrin stimulation. 24-h urinary metanephrines/ normetanephrines and serum calcium were normal.

\section{Case 3}

The patient was hypertensive with elevated 24-h urine normetadrenaline of $19703 \mathrm{mmol}(0-4900)$ and metadrenaline of $8395 \mathrm{mmol}(0-2000)$. MRI revealed a right-sided adrenal mass of $6 \times 4 \mathrm{~cm}$ consistent with phaeochromocytoma. Plasma calcitonin and serum calcium were normal.

\section{Case 4}

Phenotypic screening tests revealed an elevated basal calcitonin of $22.5 \mathrm{ng} / \mathrm{L}$, which rose to $>200 \mathrm{ng} / \mathrm{L}$ after pentagastrin stimulation. 24-h urinary metanephrines/normetanephrines and serum calcium were normal.

\section{Case 5}

Screening revealed an elevated plasma calcitonin of $28 \mathrm{ng} / \mathrm{L}$ with a pentagastrin-stimulated calcitonin level of $280 \mathrm{ng} / \mathrm{L}$. A small nodule was seen on ultrasound, and fine needle aspiration was consistent with a Thy5 lesion suggesting MTC. 24-h urinary metanephrines/ normetanephrines and serum calcium were normal.

\section{Treatment}

\section{Case 1}

The patient was stabilised on oral phenoxybenzamine therapy and a right adrenalectomy was performed.

\section{Case 2}

After discussion, he elected to have a total thyroidectomy in 2010.

\section{Case 3}

After alpha blockade, she underwent a laparoscopic rightsided adrenalectomy.

\section{Case 4}

There was no clear radiological evidence of MTC; however, she underwent a total thyroidectomy with central node dissection in 2010.

\section{Case 5}

The patient underwent a total thyroidectomy with central and left selective node dissection at levels two to five.

\section{Outcome and follow-up}

\section{Case 1}

Adrenalectomy histology showing a phaeochromocytoma. A family history revealed a son and granddaughter with a history of phaeochromocytoma, and genetic analysis revealed a C609Y mutation in the RET proto-oncogene. Subsequent blood tests revealed that basal parathyroid hormone, calcitonin and calcium levels had returned to normal without intervention. A further pentagastrin stimulation test was performed with plasma calcitonin rising from a basal value of $<4.0 \mathrm{ng} / \mathrm{L}$ to peak value of $12.7 \mathrm{ng} / \mathrm{L}(0-18.9)$, a normal response. The option of thyroid surgery was discussed with the patient, but she elected for non-surgical follow-up. Since then, fasting plasma calcitonin and serum calcium levels remain in the normal range, and there has been no evidence of recurrence or new onset of phaeochromocytoma, after six years of follow-up to date.

\section{Case 2}

Thyroidectomy histology revealed C cell hyperplasia in the right lobe, with no evidence of MTC. There has been no evidence of MTC or recurrence of phaeochromocytoma after 6 and 27 years of follow-up respectively.

\section{Case 3}

Histology revealed a phaeochromocytoma. Plasma calcitonin, serum calcium and PTH were normal. The patient tested negative for succinate dehydrogenase mutations $(S D H x)$, but RET mutational analysis was not undertaken until the following year, after the presentation of her grandmother. The patient tested positive for the same C609Y mutation found in other members of the family. Basal calcitonin was $<4.0 \mathrm{ng} / \mathrm{L}$ (normal 0-5.5) in 2009. Despite this, and after careful discussion, the patient elected for a prophylactic thyroidectomy in 2010, aged 27. Histology showed normal thyroid with no C cell hyperplasia or MTC. There is no evidence of MTC, recurrent phaeochromocytoma or hyperparathyroidism after six years of follow-up to date. 


\section{Case 4}

Histology revealed C cell hyperplasia but no MTC, with no lymph node involvement. There was an incidental finding of a $1 \mathrm{~mm}$ focus of papillary microcarcinoma. Plasma calcitonin levels have remained undetectable during six years of follow-up to date, and there has been no evidence of phaeochromocytoma during this period of follow-up. To date, her children have not consented to genetic testing.

\section{Case 5}

Histology revealed a $0.5 \mathrm{~mm}$ focus of MTC in the right lobe with no nodal metastases. Plasma calcitonin levels were $<4 \mathrm{ng} / \mathrm{L}$ on post-surgery pentagastrin stimulation tests, confirming biochemical cure of the patient's MTC, and he remains so after six years of follow-up. All plasma metanephrine testing and adrenal imaging have been negative.

The unusual history of prevalent phaeochromocytoma and lack of clinically significant MTC in our kindred prompted us to look for disease-modifying factors. Of the RET polymorphisms L769L, S836S, G691S and S904S, we identified G691S and S904S in this family.

\section{Discussion}

The RET proto-oncogene encodes for a transmembrane tyrosine kinase receptor and germline mutations are disease-causing in MEN2A (4), with different mutations having a strong genotype-phenotype correlation $(3,5)$.

Our kindred carry a mutation in codon 609 with a change from amino acid cysteine to tyrosine (C609Y). What is remarkable is that three members had an initial presentation with a phaeochromocytoma at ages 73 (Case 1), 27 (Case 2) and 25 (Case 3). These patients span three generations, and with each successive generation, there has been presentation with phaeochromocytoma at an earlier age. What is also remarkable is the low rate of MTC in this kindred (Table 1). It appears that $C$ cells in the thyroid are more susceptible to RET activation than the chromaffin cells found in the adrenal medulla (6). It has been suggested that allindividuals with MEN2A will develop MTC, with one-third developing phaeochromocytoma and one-third developing hyperparathyroidism (1). Although the penetrance of phaeochromocytoma and hyperparathyroidism is documented to be variable, there is general agreement that MTC is fully penetrant (3). Our kindred appear to have a less-aggressive thyroid phenotype and members of this family appear to have come to no harm by not having thyroidectomy at a young age. Within our kindred, four out of five of those affected with the C609Y mutation had thyroidectomies: at 27 years of age, Case 3 had a normal thyroid on histology; Cases 2 and 4 , aged 49 and 51 respectively, had $\mathrm{C}$ cell hyperplasia at the time of thyroidectomy. Cases 2, 3 and 4 comply with current thoughts on the development of MTC with progressive changes over time from normal thyroid to $\mathrm{C}$ cell hyperplasia before progression to MTC (7). However, at the age of 78, Case 1 has no biochemical evidence of C cell hyperplasia or MTC, but her grandson, Case 5, was 24 when thyroid histology revealed MTC. This family shows a lower penetrance of MTC than current literature suggests, and there is variation within the family over time of progression to MTC.

The differences in phenotype of our kindred compared to others with the C609Y gene mutation prompted us to look for other modifying factors. We found G691S and S904S RET polymorphisms that have been shown to be associated with an increased risk of developing phaeochromocytoma and a lower age of presentation in patients with other disease-causing mutations (2). We are not aware of any previous data of these polymorphisms and mutations at codon 609 , but our data suggest that such modifiers may be exerting an influence over the presentation of disease in this family, with a high rate of phaeochromocytoma and low rate of MTC. The American

Table 1 Adrenal and thyroid management of RET C609Y kindred with MEN2a.

\begin{tabular}{|c|c|c|}
\hline & Adrenal surgery & Adrenal pathology \\
\hline Case 1 & Right adrenalectomy & Phaeochromocytoma (age 73) \\
\hline Case 2 & Left adrenalectomy & Phaeochromocytoma (age 27) \\
\hline Case 3 & Right adrenalectomy & Phaeochromocytoma (age 25) \\
\hline Case 4 & None & N/A \\
\hline Case 5 & None & $\mathrm{N} / \mathrm{A}$ \\
\hline
\end{tabular}

Thyroid surgery
None
Thyroidectomy
Thyroidectomy
Thyroidectomy and central node
dissection
Thyroidectomy and central node
dissection

\begin{tabular}{l} 
Thyroid pathology \\
\hline N/A \\
C cell hyperplasia (aged 49) \\
Normal (aged 27) \\
C cell hyperplasia (aged 51 )
\end{tabular}

MTC (aged 24)

MTC, medullary thyroid cancer; N/A, not applicable. 
Thyroid Association (ATA) and 2014 British Thyroid Association Guidelines for the Management of Thyroid Cancer suggest prophylactic thyroid surgery at the age of 5 be discussed with the parents of patients with moderaterisk mutations (ATA-MOD), including those with $\Delta 609$ mutations $(8,9)$. It is recommended that surgery may be delayed beyond 5 years of age if the following criteria are met: normal serum calcitonin, normal neck ultrasound, a less aggressive MTC family history and family preference (8). Due to such a low rate of MTC in our kindred, it is possible that other kindreds harbouring this mutation might not need early thyroid surgery and that the polymorphisms identified here in association with C609Y might be contributing to a protective phenotype for the development of MTC.

For those with 609 mutations, screening for phaeochromocytoma should commence at the age of 16 according to the ATA guidelines and at the age of 8 according to the European guidelines $(8,10)$. The youngest member of our kindred to present with phaeochromocytoma was 25 years of age. Nevertheless, the presentation of phaeochromocytoma in Case 1 at age 73 emphasises the need for life-long surveillance in MEN2A families with this mutation. In contrast, Calva et al. screened 83 patients with the C609Y mutation and found no evidence of phaeochromocytoma, concluding that patients with this mutation may not need to be screened for this complication (1). Our data differ from these recommendations, and as phaeochromocytoma is a potentially life-threatening condition, we highlight the importance of life-long screening for this condition in patients with MEN2A.

In summary, C609Y RET mutations may be associated with a life-long risk of phaeochromocytoma and a lower risk of MTC than often quoted. There may be a role for the routine screening of RET polymorphisms, and this is greatly facilitated by the increasing ease of access to nextgeneration sequencing.

\section{Declaration of interest}

The authors declare that there is no conflict of interest that could be perceived as prejudicing the impartiality of the research reported.

\section{Funding}

This research did not receive any specific grant from any funding agency in the public, commercial or not-for-profit sector.
Patient consent

Written, informed consent has been received from the patients.

\section{Author contribution statement}

Rowena Speak wrote the case series and performed literature review. Dr Jackie Cook performed genetic analysis and co-wrote the report. Mr Barney Harrison performed endocrine surgery (thyroid and adrenal) on patients and co-wrote the report. Prof John Newell-Price is the physician responsible for the patients and designed and co-wrote the report.

\section{Acknowledgements}

The authors would like to thank the family members and the staff on the Endocrine Unit at the Royal Hallamshire Hospital in Sheffield.

\section{References}

1 Calva D, O’Dorisio TM, Sue O’Dorisio M, Lal G, Sugg S, Weigel RJ \& Howe JR 2009 When is prophylactic thyroidectomy indicated for patients with the RET codon 609 mutation? Annals of Surgical Oncology 16 2237-2244. (doi:10.1245/s10434-009-0524-3)

2 Siqueira DR, Ceolin L, Ferreira CV, Romitti M, Maia SC, Maciel LM \& Maia AL 2014 Role of RET genetic variants in MEN2-associated phaeochromocytoma. European Journal of Endocrinology 170 821-828. (doi:10.1530/EJE-14-0084)

3 Mian C, Sartorato P, Barollo S, Zane M \& Opocher G 2012 RET codon 609 mutations: a contribution for better clinical managing. Clinics 67 (Supplement 1) 33-36. (doi:10.6061/clinics/2012(Sup01)07)

4 Mulligan LM, Kwok JB, Healey CS, Elsdon MJ, Eng C, Gardner E, Love DR, Mole SE, Moore JK, Papi L, et al. 1993 Germ-line mutations of the RET proto-oncogene in multiple endocrine neoplasia type $2 \mathrm{~A}$. Nature 363 458-460. (doi:10.1038/363458a0)

5 Yip L, Cote GJ, Shapiro SE, Ayers GD, Herzog CE, Sellin RV Sherman SI, Gagel RF, Lee JE \& Evans DB 2003 Multiple endocrine neoplasia type 2: evaluation of the genotype-phenotype relationship. Archives of Surgery 138 409-416; discussion 416. (doi:10.1001/ archsurg.138.4.409)

6 Jhiang SM 2000 The RET proto-oncogene in human cancers. Oncogene 19 5590-5597. (doi:10.1038/sj.onc.1203857)

7 Machens A, Lorenz K, Sekulla C, Hoppner W, Frank-Raue K, Raue F \& Dralle H 2013 Molecular epidemiology of multiple endocrine neoplasia 2: implications for RET screening in the new millenium. European Journal of Endocrinology 168 307-314. (doi:10.1530/EJE-120919)

8 Wells SA Jr, Asa SL, Dralle H, Elisei R, Evans DB, Gagel RF, Lee N, Machens A, Moley JF, Pacini F, et al. 2015 Revised american thyroid association guidelines for the management of medullary thyroid carcinoma. Thyroid 25 567-610. (doi:10.1089/thy.2014.0335)

9 Perros P, Boelaert K, Colley S, Evans C, Evans RM, Gerrard Ba G, Gilbert J, Harrison B, Johnson SJ, Giles TE, et al. 2014 Guidelines for the management of thyroid cancer. Clinical Endocrinology $\mathbf{8 1}$ (Supplement 1) 1-122. (doi:10.1111/cen.12515)

10 Elisei R, Alevizaki M, Conte-Devolx B, Frank-Raue K, Leite V \& Williams GR 20132012 European thyroid association guidelines for genetic testing and its clinical consequences in medullary thyroid cancer. European Thyroid Journal 1 216-231. (doi:10.1159/000346174)

Received in final form 28 October 2016

Accepted 7 November 2016 